\title{
Human-Induced Pluripotent Stem Cell-Derived Cardiomyocytes Platform to Study SARS-CoV-2 Related Myocardial Injury
}

\author{
Chun-Ka Wong; Hayes Kam-Hei Luk, PhD; Wing-Hon Lai, PhD; Yee-Man Lau, PhD; \\ Ricky Ruiqi Zhang, PhD; Antonio Cheuk-Pui Wong; George Chi-Shing Lo, PhD; \\ Kwok-Hung Chan, PhD; Ivan Fan-Ngai Hung, MD; Hung-Fat Tse, MD, PhD; \\ Patrick Chiu-Yat Woo, MD; Susanna Kar-Pui Lau, MD; Chung-Wah Siu, MD
}

Background: SARS-CoV-2 infection is associated with myocardial injury, but there is a paucity of experimental platforms for the condition.

\begin{abstract}
Methods and Results: Human-induced pluripotent stem cell-derived cardiomyocytes (hiPSC-CMs) infected by SARS-CoV-2 for 3 days ceased beating and exhibited cytopathogenic changes with reduced viability. Active viral replication was evidenced by an increase in supernatant SARS-CoV-2 and the presence of SARS-CoV-2 nucleocaspid protein within hiPSC-CMs. Expressions of BNP, CXCL1, CXCL2, IL-6, IL-8 and TNF- $\alpha$ were upregulated, while ACE2 was downregulated.
\end{abstract}

Conclusions: Our hiPSC-CM-based in-vitro SARS-CoV-2 myocarditis model recapitulated the cytopathogenic effects and cytokine/ chemokine response. It could be exploited as a drug screening platform.

Key Words: COVID-19; Myocarditis; SARS-CoV-2; Stem cells

C oronavirus disease 2019 (COVID-19) caused by severe acute respiratory syndrome coronavirus 2 (SARS-CoV-2) emerged in December 2019 and resulted in a pandemic of viral pneumonia. Although the virus primarily affects the respiratory system, cardiac involvement has been commonly observed. In early case series, up to $27.8 \%$ of hospitalized COVID-19 patients had elevated troponin levels, indicating myocardial injury, which is substantially higher than with other common respiratory viral illnesses such as influenza. ${ }^{1}$ Plausibly, COVID-19 may trigger acute coronary events in patients with coronary artery disease (CAD) as in other viral illnesses. However, many COVID-19 patients with myocardial injury did not have underlying CAD. Furthermore, there have been case series reporting SARS-CoV-2-related fulminant myocarditis. ${ }^{2}$ These findings suggest possible alternative pathogenic mechanisms such as a direct cytopathogenic effect of SARS-CoV-2 on human cardiomyocytes (CMs). Indeed, such a hypothesis is supported by several well- established facts. First, angiotensin-converting enzyme 2 (ACE2), the functional receptor for SARS-CoV-2, is abundantly expressed in CMs. ${ }^{3}$ Second, SARS-CoV-2 viremia has been observed in up to $22 \%$ of COVID-19 patients, providing a possible portal for the virus to spread from the respiratory system to the myocardium. ${ }^{4}$

As COVID-19 patients with myocardial injury typically have higher mortality and morbidity rates, ${ }^{1}$ it is of clinical importance to elucidate the underlying pathogenic mechanisms. However, this is limited by the paucity of experimental platforms for studying the process. For instance, the golden Syrian hamster, a commonly used animal model for SARS-CoV-2 infection, has not demonstrated SARSCoV-2-associated myocarditis. 5 Theoretically, direct characterization of myocardial tissues obtained from patients with SARS-CoV-2-related myocardial injury could provide the much-needed insight regarding its underlying pathogenesis, but it is limited by technical difficulties as well as obvious ethical concerns. More importantly, adult

Received August 24, 2020; revised manuscript received September 3, 2020; accepted September 9, 2020; J-STAGE Advance Publication released online September 26, 2020 Time for primary review: 6 days

Cardiology Division, Department of Medicine (C.-K.W., W.-H.L., Y.-M.L., H.-F.T., C.-W.S.), Department of Microbiology (H.K.-H.L., A.C.-P.W., G.C.-S.L., P.C.-Y.W., S.K.-P.L.), Infectious Disease Division, Department of Medicine (R.R.Z., K.-H.C., I.F.-N.H.), Li Ka Shing Faculty of Medicine, The University of Hong Kong, Hong Kong SAR, China

C.-K.W. and H.K.-H.L. are co-first authors.

C.-W.S. and S.K.-P.L. are co-senior and corresponding authors.

Mailing address: Chung-Wah Siu, MD, Cardiology Division, Department of Medicine, Li Ka Shing Faculty of Medicine, The University of Hong Kong, Hong Kong SAR, China. E-mail: cwdsiu@hku.hk and Susanna Kar-Pui Lau, MD, Department of Microbiology, The University of Hong Kong, Hong Kong SAR, China. E-mail: skplau@hku.hk

All rights are reserved to the Japanese Circulation Society. For permissions, please e-mail: cj@j-circ.or.jp

ISSN-1346-9843 
human CMs do not readily proliferate in vitro, precluding further experimentation such as drug screening. The ability to generate human-induced pluripotent stem cells (hiPSCs) and their cardiac derivatives offers an opportunity to study cardiac diseases in vitro. Thus, we exploited our previously established hiPSC-CMs to study SARS-CoV-2 susceptibility and possible cytopathogenic effects. In addition to providing direct experimental evidence of SARS-CoV-2-related myocardial injury, the hiPSC-CM platform could also be exploited as a drug screening platform for COVID-19.

\section{Methods}

\section{hiPSC Generation, Characterization, and In Vitro Cardiac Differentiation}

The study protocol for procurement of human tissue for the generation of hiPSCs was approved by the Institutional Review Board of the University of Hong Kong/Hospital Authority Hong Kong West Cluster (HKU/HA HKW IRB) and was registered at the Clinical Trial Center, the University of Hong Kong (IRB-UW08-258). In this study, the hiPSCs used were derived from a healthy Chinese man with no known cardiac disease and who gave written informed consent. Detailed methods of hiPSC generation and characterization have been previously reported by our group. ${ }^{6}$ Skin biopsies were mechanically dissociated, plated and cultured with $10 \%$ fetal bovine serum. Dermal fibroblasts growing out from the skin tissues were expanded and transduced with lentiviruses encoding OCT4, SOX2, KLF4, and c-MYC. Putative hiPSC clusters were typically observed 14-21 days after lentiviral transduction, and were manually dissected and expanded in 6-well plates precoated with Matrigel (Thermo Scientific Inc., USA). The authenticity of the hiPSCs was confirmed by expression of a panel of pluripotent markers including OCT4, Nanog, KLF, SSEA-4, TRA-1-60, and SOX2, exogenous transgene silencing (OCT4 and Nanog), OCT4 promoter demethylation, and teratoma formation after inoculation into severe combined immunodeficiency mice as previously reported. ${ }^{7}$

Cardiac differentiation was induced as previously reported. ${ }^{6}$ Briefly, undifferentiated hiPSCs were maintained in mTeSRTM1 medium (STEMCELL Technologies Inc., Canada). At 4 days before induction, the hiPSCs were dissociated into single cells with accutase (Invitrogen, USA) and then seeded into a 12-well Matrigel-coated plate supplemented with Y27632 (5 $\mu \mathrm{mol} / \mathrm{L})$ (Stemgent, USA). On the first induction day the culture medium was switched to RPMI medium (Life Technologies, USA) without insulin and supplemented with B27 (Life Technologies) and a GSK-b inhibitor, CHIR99021 (12 $\mu \mathrm{mol} / \mathrm{L})$ (Selleckchem, USA), and refreshed $24 \mathrm{~h}$ later. On day 4 , a Wnt signaling inhibitor, IWP2 $(5 \mu \mathrm{mol} / \mathrm{L})$ (Selleckchem), was added to the culture medium. Spontaneously beating CMs were typically observed around 9 days after induction. The cells were maintained in $\mathrm{CM}$ maintenance medium (RPMI medium with B27 supplement). Standard immunofluorescent staining with $\alpha$-actinin and electrophysiology analysis by patch clamping were performed to confirm their cardiac phenotype.

\section{SARS-CoV-2 Preparation and Infection}

All experiments involving live SARS-CoV-2 were performed according to the approved standard operating procedures of the HKU Biosafety Level-3 facility. The collection and use of clinical samples and data were approved by HKU/HA HKW IRB (UW 16-365 20-072016). SARS-CoV-2 was isolated from the nasopharyngeal swab specimens of SARS-CoV-2 infected patients in Hong Kong with Vero cells and passaged twice. The plaquepurified viral isolate was amplified by an additional passage in Vero cells to make working stocks of the virus. At 28 days after induced cardiac differentiation, the hiPSC$\mathrm{CMs}$ were dissociated into a single-cell suspension. Approximately $3 \times 10^{4}$ and $1 \times 10^{4}$ hiPSC-CMs were then replated into 24-well and 96-well culture dishes, respectively, precoated with Matrigel. SARS-CoV-2 were applied to monolayers of hiPSC-CMs with multiplicity of infection (MOI) of 0.1 or 1 , depending on experimental allocation, and incubated at $37^{\circ} \mathrm{C}$ for $1 \mathrm{~h}$.

\section{Immunofluorescence Staining}

The hiPSC-CMs were dissociated by collagenase treatment and seeded on gelatin-coated cover-slips for immunofluorescence studies. Next, they were fixed, permeabilized with $4 \%$ paraformaldehyde for $1 \mathrm{~h}$, and washed with Dulbecco phosphate-buffered saline with $0.1 \%$ Triton X-100 (SigmaAldrich, USA). Immunofluorescence staining of SARSCoV-2 was performed with rabbit anti-SARS-CoV-2 nucleocaspid protein (NP) primary antibody and fluorescein isothiocyanate (FITC)-conjugated goat-anti-rabbit antibody. Antibodies against ACE2 (R\&D Biosystem, USA) and DAPI for nuclei staining were applied. Images were acquired using a fluorescence confocal Carl Zeiss LSM 700 microscope (Zeiss GmbH, Germany).

\section{Viability Assay}

The viability of the hiPSC-CMs was evaluated using Cell Counting Kit-8 (CCK-8; Dojindo Molecular Technologies Inc., USA). After SARS-CoV-2 infection, $10 \mu \mathrm{L}$ of CCK-8 reagent was added to the hiPSC-CMs and allowed to react for $2 \mathrm{~h}$. The absorbance measurement at $450 \mathrm{~nm}$ was measured for cell viability, expressed in percentage.

\section{Reverse Transcription Quantitative PCR (RT-qPCR)}

Supernatant RNA was extracted using a Viral RNA Mini Kit (Qiagen, Germany) for RT-qPCR targeting the envelope gene of SARS-CoV-2. Cell lysate RNA was extracted using an RNeasy Mini Kit (Qiagen, Germany) for RTqPCR targeting B-type natriuretic peptide (BNP), a cytokine/chemokine panel and ACE2 using troponin T as the reference gene for normalization.

\section{Statistical Analysis}

Continuous variables are expressed as mean \pm SEM. Statistical comparisons between groups were performed using Student's t-test or nonparametric Mann-Whitney test. Variables involving more than 2 groups were analyzed using one-way ANOVA. Calculations were performed with GraphPad Prism 5 (GraphPad). A P value $<0.05$ was considered statistically significant and will be annotated with asterisk $(*)$.

\section{Results}

Direct Cytopathogenic Effects of SARS-CoV-2 on hiPSC-CMs To recapitulate the SARS-CoV-2-associated myocardial injury, hiPSC-CMs were infected with SARS-CoV-2 for 3 days. Under brightfield microscopy, the monolayers of hiPSC-CMs ceased spontaneous beating, while mock hiPSC-CMs continued to contract. Figure A shows repre- 


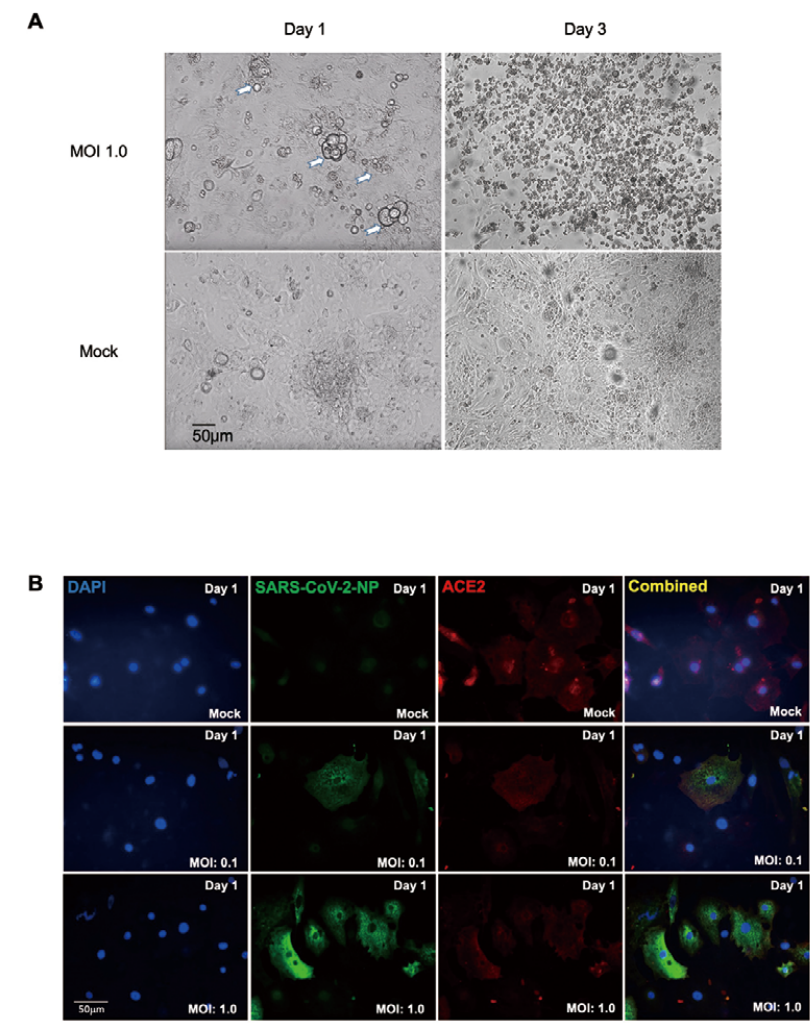

D

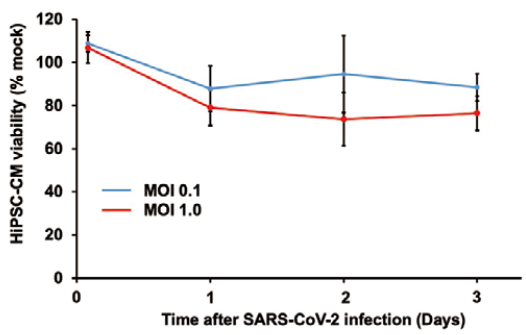

E

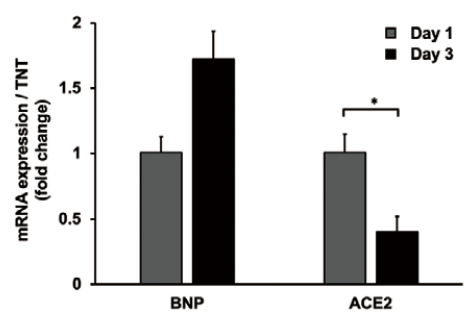

$\mathbf{F}$

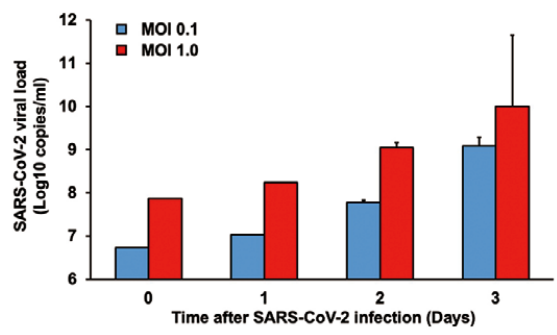

C
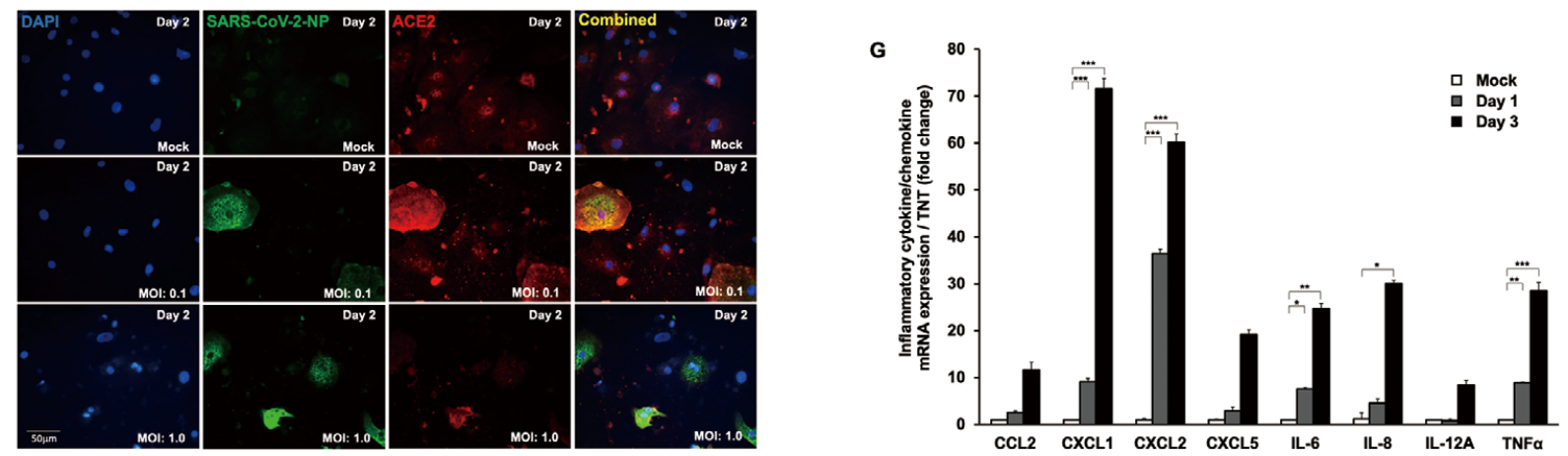

Figure. Human-induced pluripotent stem cell-derived cardiomyocytes (hiPSC-CMs) platform to study SARS-CoV-2-related myocardial injury. (A) Brightfield microscopy showing typical cytopathogenic changes on day 1 and day 3 after SAR-CoV-2 infection at $\mathrm{MOI}$ of 1 (arrows: cell rounding and clumping together in "grape-like" clusters). (B,C) Representative immunofluorescence staining of hiPSC-CMs (red: ACE2, green: SARS-CoV-2-NP; blue: DAPI) on day 1 and day 2 after SARS-CoV-2 infection at MOI of 0.1 and 1. (D) Colorimetric assays (CCK-8) demonstrated reduced hiPSC-CM viability after SARS-CoV-2 infection. (E) Relative mRNA expression of ACE2 and BNP in cell lysate of hiPSC-CMs by RT-qPCR with TNT as internal control ( $\left.{ }^{*} P<0.05\right)$. (F) RT-qPCR for SARS-CoV-2 RNA in the supernatant of hiPSC-CMs after SARS-CoV-2 infection and (G) relative mRNA expression of cytokines/ chemokines in cell lysate of hiPSC-CMs by RT-qPCR with TNT as internal control $\left({ }^{\star} P<0.05,{ }^{*} P<0.01\right.$ and $\left.{ }^{* \star *} P<0.001\right)$. ACE, angiotensin-converting enzyme; BNP, B-type natriuretic peptide; MOI, multiplicity of infection; NP, nucleocaspid protein; RT-qPCR, reverse transcription quantitative polymerase chain reaction; SARS-CoV-2, severe acute respiratory syndrome coronavirus 2; TNT, troponin $\mathrm{T}$.

sentative brightfield microscopy images of hiPSC-CMs at 1 and 3 days after SARS-CoV-2 infection at MOI of 1 . The hiPSC-CMs infected with SARS-CoV-2 exhibited typical cytopathogenic changes with cell rounding and clumping together in "grape-like" clusters on day 1 and near total destruction of the hiPSC-CMs monolayer with pyknotic shrinking and detachment from the culture dish on day 3 . Figure B,C show representative immunofluorescence staining of hiPSC-CMs under mock conditions of SARS-CoV-2 infection at MOI of 0.1 and 1 on days 1 and 2, respectively. SARS-CoV-2 NP (green) was detected within hiPSC-CMs infected at MOI of 0.1 and more robustly within hiPSC- 
CMs infected at MOI of 1 since day 1, with increase in intensity on day 2. At the same time, cell shrinkage and deformation, indicating direct cytopathogenic effects, were observed, particularly in hiPSC-CMs infected with SARSCoV-2 at MOI of 1 on day 2 (Figure B,C). Consistently, the $\mathrm{CCK} 8$ assay demonstrated a dose-dependent reduction in hiPSC-CM viability after SARS-CoV-2 infection, with mean absorbance measurement at $450 \mathrm{~nm}$ reduced from $108.8 \pm 2.2 \%$ to $88.5 \pm 3.7 \%$ of mock hiPSC-CMs infected at MOI of $0.1\left(\mathrm{P}=0.009^{*}\right)$ and from $106.8 \pm 4.2 \%$ to $76.5 \pm 4.6 \%$ of mock hiPSC-CMs infected at MOI of $1\left(\mathrm{P}=0.008^{*}\right)$ (Figure D). In addition, SARS-CoV-2 infection at MOI of 1 increased the mRNA expression of BNP by 1.72 -fold $(\mathrm{P}=0.089)$, but reduced the mRNA expression of ACE2 by 0.39 -fold $\left(\mathrm{P}<0.05^{*}\right)$ in hiPSC-CMs (Figure E).

\section{Active SARS-CoV-2 Replication in hiPSC-CMs}

To ascertain active viral replication, supernatant from the hiPSC-CMs was collected after SARS-CoV-2 infection for RT-qRCR of SARS-CoV-2 RNA from day 0 to day 3 (Figure F). A progressive increase in SARS-CoV-2 RNA in the supernatant was observed, with 2.66 and $2.13 \log 10$ copies/mL increase in hiPSC-CMs infected at MOI of 0.1 and 1 , respectively, indicating active viral replication comparable to other human cell lines.

\section{Cytokine/Chemokine Activation in hiPSC-CMs With SARS-CoV-2 Infection}

In addition to direct cytopathogenic effects, SARS-CoV-2 infection may cause myocardial injury through cardiac inflammation. To study the inflammatory response of hiPSC-CMs after SARS-CoV-2 infection, the mRNA expressions of 8 inflammatory cytokines/chemokines were quantified with RT-qPCR using cell lysate from hiPSCCMs infected at MOI of 1 at 2 and $72 \mathrm{~h}$ after infection (Figure G). Among 5 inflammatory cytokines/chemokines (chemokine (C-X-C motif) ligand (CXCL) 1, CXCL2, interleukin (IL)-6, IL-8 and tumor necrosis factor (TNF)- $\alpha$ ) showing a significant increase in expression after SARSCoV-2 infection, CXCL1 and CXCL2 were the most markedly upregulated, with 9.1-fold and 36.5-fold increments, respectively $\left(\mathrm{P}<0.001^{*}\right) 2 \mathrm{~h}$ after infection, and 71.5 fold and 60.2-fold increments, respectively $\left(\mathrm{P}<0.001^{*}\right) 72 \mathrm{~h}$ after infection.

\section{Discussion}

In the present study, we exploited hiPSC-CMs to provide direct experimental evidence of the susceptibility of human CMs to SARS-CoV-2, and to elucidate the mechanisms underlying SARS-CoV-2-induced myocardial injury. These include direct viral cytopathogenic effects and upregulation of inflammatory cytokines/chemokines. More importantly, our hiPSC-CM platform could potentially be used to assess responsiveness of novel pharmacological agents for SARS-CoV-2.

Unlike other respiratory viruses, SARS-CoV-2 is unique for causing myocardial injury in an unusually high proportion of patients. ${ }^{1}$ Such a high prevalence of myocardial injury has not been observed even in outbreaks of more virulent human coronaviruses such as SARS-CoV and MERS-CoV. More importantly, the presence of myocardial injury confers worse outcomes in COVID-19 patients. Despite the paucity of direct experimental evidence, it has been hypothesized that SARS-CoV-2-related myocardial injury is a result of direct viral cytopathogenic effects, host immune response, or a combination of both. It was confirmed in the present study that SARS-CoV-2 is capable of infecting and replicating in human CMs. Our results also showed that SARS-CoV-2 infection adversely affects the cellular function of human CMs, by suppressing ACE2 expression and increasing BNP expression. It is well known that ACE2 exerts cardioprotective effects through conversion of the vasoconstrictive and proliferative peptide angiotensin II to the vasodilatory and antiproliferative peptide angiotensin-(1-7) in the ACE2-angiotensin-(1-7)-Mas axis. Pharmacological agents for the treatment of heart failure, including ACE inhibitors, angiotensin II receptor blockers, and mineralocorticoid antagonists, enhance the cardiac expression of ACE2 to protect the heart from angiotensin II overload. In the context of coronavirus infection, it has been shown that the SARS-CoV spike protein triggered translocation of ACE2 could lead to downregulation of ACE2 transcription. ${ }^{8}$ This is in concordance with the clinical observation that ACE2 mRNA and protein expression are reduced in the myocardium of patients who died of SARS-CoV infection in 2003. ${ }^{9}$ Coronavirus-induced downregulation of cardiac ACE2 expression may lead to an imbalance of angiotensin II over-activity and antiotensin-1-7 deficiency, which triggers inflammation, thrombosis and other adverse reactions. In addition to direct cytopathogenic effects, SARS-CoV-2 infection also results in upregulation of a panel of inflammatory cytokines/chemokines, including CXCL1, CXCL2, IL-6, IL-8 and TNF. Of note, CXCL1 and its receptor CXCR2 have been shown to play a critical role in mediating angiotensin IIinduced monocyte infiltration to the heart. ${ }^{10}$ This indicates that the host immune response may also play a role in SARS-CoV-2-related myocardial injury.

SARS-CoV-2 has resulted in a pandemic with over 30 million infections and 800,000 deaths globally, and yet there is no sign of when the pandemic will actually end. Thus an increasing number of patients with SARS-CoV2-related myocarditis can be anticipated. In the present study, we demonstrated that our hiPSC-CM platform can be used to recapitulate SARS-CoV-2-related myocardial injury, ranging from susceptibility, to direct cytopathogenic effects and inflammatory cytokine/chemokine response. The platform could be further exploited to study pathogenic mechanisms and to identify effective pharmacological agents such as antiviral medications and immunomodulatory therapies.

\section{Study Limitations}

First, although our hiPSC-CM platform successfully recapitulated the direct cytopathogenic effects of SARS-CoV-2 infection, the host immune response, including inflammatory cell infiltration in the setting of myocarditis, could not be fully elucidated. Second, detailed cellular functional assessment of SARS-CoV-2-infected hiPSC-CMs, including cellular electrophysiology, calcium handling properties, and contractile function, could not be performed because of the strict restriction of live SARS-CoV-2-infected cells outside the Biosafety Level-3 facility.

\section{Conclusions}

A hiPSC-CM platform recapitulated SARS-CoV-2-induced myocardial injury and could be further exploited to study the underlying pathogenic mechanisms and pharma- 
cotherapeutic development.

\section{Acknowledgments}

This work was partly supported by the Theme-based Research Scheme (Project no. T11-707/15-R), University Grant Committee; University Development Fund, The University of Hong Kong, Hong Kong SAR, China; and the Collaborative Innovation Center for Diagnosis and Treatment of Infectious Diseases, Ministry of Education, China.

\section{Disclosures}

The authors declare no conflicts of interest.

\section{Ethics Approval}

Institutional Review Board of the University of Hong Kong/Hospital Authority Hong Kong West Cluster, Hong Kong SAR, China (IRBUW08-258 and UW 16-365 20-07-2016).

\section{References}

1. Guo T, Fan Y, Chen M, Wu X, Zhang L, He T, et al. Cardiovascular implications of fatal outcomes of patients with coronavirus disease 2019 (COVID-19). JAMA Cardiol 2020; 5: 811-818.

2. Trogen B, Gonzalez FJ, Shust GF. COVID-19-associated myocarditis in an adolescent. Pediatr Infect Dis $J$ 2020; 39: e204-e205.

3. Liu H, Gai S, Wang X, Zeng J, Sun C, Zhao Y, et al. Single-cell analysis of SARS-CoV-2 receptor ACE2 and spike protein prim- ing expression of proteases in the human heart. Cardiovasc Res 2020; 116: $1733-1741$.

4. To KK, Tsang OT, Leung WS, Tam AR, Wu TC, Lung DC, et al. Temporal profiles of viral load in posterior oropharyngeal saliva samples and serum antibody responses during infection by SARS-CoV-2: An observational cohort study. Lancet Infect Dis 2020; 20: $565-574$

5. Chan JF, Zhang AJ, Yuan S, Poon VK, Chan CC, Lee AC, et al. Simulation of the clinical and pathological manifestations of Coronavirus Disease 2019 (COVID-19) in golden Syrian hamster model: Implications for disease pathogenesis and transmissibility. Clin Infect Dis, doi:10.1093/cid/ciaa325.

6. Lee YK, Lau YM, Cai ZJ, Lai WH, Wong LY, Tse HF, et al. Modeling treatment response for lamin A/C related dilated cardiomyopathy in human induced pluripotent stem cells. $J \mathrm{Am}$ Heart Assoc 2017; 6: e005677.

7. Lai WH, Ho JC, Lee YK, Ng KM, Au KW, Chan YC, et al. ROCK inhibition facilitates the generation of human-induced pluripotent stem cells in a defined, feeder-, and serum-free system. Cell Reprogram 2010; 12: 641-653.

8. Kuba K, Imai Y, Rao S, Gao H, Guo F, Guan B, et al. A crucial role of angiotensin converting enzyme 2 (ACE2) in SARS coronavirus-induced lung injury. Nat Med 2005; 11: 875-879.

9. Oudit GY, Kassiri Z, Jiang C, Liu PP, Poutanen SM, Penninger $\mathrm{JM}$, et al. SARS-coronavirus modulation of myocardial ACE2 expression and inflammation in patients with SARS. Eur J Clin Invest 2009; 39: 618-625.

10. Wang L, Zhang YL, Lin QY, Liu Y, Guan XM, Ma XL, et al. CXCL1-CXCR2 axis mediates angiotensin II-induced cardiac hypertrophy and remodelling through regulation of monocyte infiltration. Eur Heart J 2018; 39: 1818-1831. 